\title{
Calcified Amorphous Tumor in the Left Atrium in a Patient on Long-term Peritoneal Dialysis
}

\author{
Akihito Tanaka $^{1}$, Masashi Mizuno ${ }^{1,2}$, Yasuhiro Suzuki ${ }^{1,2}$, Hideki Oshima ${ }^{3}$, \\ Fumiko Sakata $^{1,2}$, Hideaki Ishikawa ${ }^{4}$, Saori Tsukushi ${ }^{4}$ and Yasuhiko Ito ${ }^{1,2}$
}

\begin{abstract}
A 66-year-old woman with an 11-year history of peritoneal dialysis (PD) for diabetic nephropathy and renal failure exhibited a movable tumor in the left atrium on echocardiography. Tumor resection was performed due to the difficulty in diagnosing the tumor and the future risk of heart failure and embolization. Light microscopy showed a calcified amorphous tumor (CAT), a rare intracardiac mass characterized by the presence of a pedicle and diffuse calcification. An increased calcium-phosphate product level was suspected as an etiology, although degeneration, inflammation and/or mineral balance disorders may also induce the development of CAT. We herein report the first known case of CAT in a PD patient.
\end{abstract}

Key words: calcified amorphous tumor, peritoneal dialysis, bone mineral disease

(Intern Med 54: 481-485, 2015)

(DOI: 10.2169/internalmedicine.54.2967)

\section{Introduction}

A calcified amorphous tumor (CAT) is a non-neoplastic cardiac tumor characterized by the presence of a pedicle and diffuse calcification that was originally described in 1997 (1). The pathological features of CAT include acellular/hypocellular tumor components with abundant fibrous tissue and nodular calcium deposits (1). Although the etiology remains unclear, degeneration, focal chronic inflammation and/or mineral balance disorders may be involved. Since its original description, a few cases of CAT have been reported in patients with end-stage renal disease (ESRD) under hemodialysis (HD) (2-6). However, no reports have previously described CAT in patients on peritoneal dialysis (PD). We herein report the first case of CAT in a PD patient.

\section{Case Report}

A 66-year-old woman with ESRD secondary to diabetic nephropathy was referred to our hospital. She had been maintained on PD for the past 11 years and had since experienced two episodes of PD-related peritonitis, at nine years and three months prior to the current admission. Other than these episodes, no marked infectious events had been noted, including periodontitis, dental caries or episodes of bacteremia. The patient had started PD with a prescription comprising $1.5 \mathrm{~L}$ of $1.5 \%$ glucose solution (Dianeal-N PD-2 $1.5 \%^{\mathrm{TM}}$, Baxter, Tokyo, Japan; Table 1) three times in the daytime and once overnight, with good control of calcium and phosphate metabolism as well as body fluids. In 2007, a decreased urine volume resulted in volume overload, and the PD recipe was changed three times (Fig. 1). Finally, the PD regimen was as follows: $1.5 \mathrm{~L}$ of $2.5 \%$ glucose solution (Dianeal-N PD-2 2.5\% ${ }^{\mathrm{TM}}$, Baxter) three times in the daytime and $1.5 \mathrm{~L}$ of icodextrin solution overnight. The dialysate-toplasma creatinine concentration ratio (D/P creatinine) on peritoneal equilibration tests and the efficacy of dialysis (weekly creatinine clearance and $\mathrm{Kt} / \mathrm{V}$ ) are summarized in Table 2. Despite our best efforts, it was difficult to control the phosphate level, and the amount of oral phosphate binder, including calcium carbonate, was increased until 2010 (Fig. 1). In January 2009, the administration of ci-

\footnotetext{
${ }^{1}$ Division of Nephrology, Nagoya University Graduate School of Medicine, Japan, ${ }^{2}$ Renal Replacement Therapy, Nagoya University Graduate School of Medicine, Japan, ${ }^{3}$ Division of Cardiac Surgery, Nagoya University Graduate School of Medicine, Japan and ${ }^{4}$ Division of Nephrology, Tokai Chuo Hospital, Japan

Received for publication March 26, 2014; Accepted for publication July 14, 2014

Correspondence to Dr. Masashi Mizuno,mmizu@med.nagoya-u.ac.jp and masashim1jp@yahoo.co.jp
} 
Table 1. Prescription Medication and Ionic Concentration of Peritoneal Dialysis (PD) Solution on the Day of Admission

\begin{tabular}{|c|c|c|}
\hline \multicolumn{3}{|l|}{ Medication } \\
\hline \multicolumn{2}{|c|}{ Cinacalcet hydrochloride } & $25 \mathrm{mg} / 2$ days \\
\hline \multicolumn{2}{|c|}{ Alfacalcidol } & $0.25 \mu \mathrm{g} /$ day \\
\hline \multicolumn{2}{|l|}{ Calcium carbonate } & $1,500 \mathrm{mg} /$ day \\
\hline \multicolumn{2}{|c|}{ Sevelamer hydrochloride } & $2,250 \mathrm{mg} /$ day \\
\hline \multicolumn{2}{|l|}{ Nifedipine } & $40 \mathrm{mg} /$ day \\
\hline \multicolumn{2}{|l|}{ Telmisartan } & $80 \mathrm{mg} /$ day \\
\hline \multicolumn{2}{|l|}{ Vildagliptin } & $50 \mathrm{mg} /$ day \\
\hline PD solution & $\begin{array}{l}\text { Dianeal-N PD-2 } \\
\text { (glucose solution) }\end{array}$ & $\begin{array}{l}\text { Extraneal } \\
\text { (icodextrin solution) }\end{array}$ \\
\hline $\mathrm{Na}^{+}(\mathrm{mEq} / \mathrm{L})$ & 132 & 132 \\
\hline $\mathrm{Ca}^{2+}(\mathrm{mEq} / \mathrm{L})$ & 3.5 & 3.5 \\
\hline $\mathrm{Mg}^{2+}(\mathrm{mEq} / \mathrm{L})$ & 0.5 & 0.5 \\
\hline $\mathrm{Cl}^{-}(\mathrm{mEq} / \mathrm{L})$ & 96 & 96 \\
\hline Lactate $(\mathrm{mEq} / \mathrm{L})$ & 40 & 40 \\
\hline
\end{tabular}

nacalcet hydrochloride was started due to a persistently high serum level of intact parathyroid hormone (iPTH). In 2008, echocardiography was performed to assess the cardiac function (white arrow in Fig. 1), which was found to be within the normal range, with no apparent intracardiac masses. However, high serum levels of corrected calcium and phosphate continued for approximately three years, from 2007 to 2010 (Fig. 1). The corrected calcium-phosphate product level was largely maintained at less than $50 \mathrm{mg}^{2} / \mathrm{dL}^{2}$ until 2007 then subsequently increased, reaching $>70 \mathrm{mg}^{2} / \mathrm{dL}^{2}$ in 2009 and 2010. This exacerbation appeared to be caused by a decrease in the urine volume as well as poor control of the patient's diet. In 2011, a routine echocardiography scan (black arrow in Fig. 1) disclosed a hyperechoic movable mass adhering to the mitral valve. We recommended surgical treatment based on a perceived risk of embolism $(7,8)$; however, the patient initially declined to undergo surgical intervention since she remained asymptomatic. Following our repeated recommendations, she finally consented to surgery and was referred to our hospital. Due to her long history of $\mathrm{PD}$, an arteriovenous fistula on the left forearm was created prior to referral to our hospital. However, the patient had no other past medial history or history of anticoagulant or antiplatelet treatment. The hemoglobin A1c level [National glycohemoglobin standardization program (NGSP) value] had been largely controlled between $6.0 \%$ and $7.0 \%$ until a few months before admission for resection of the cardiac tumor. The details of the medications prescribed on referral to our hospital are shown in Table 1.

At the time of referral to our hospital, the patient remained asymptomatic. On admission, a physical examination revealed clear respiratory sounds and a slight cardiac murmur during systole on chest auscultation (Levine II/IV around the intersection of the midclavicular line and fifth intercostal space). The abdomen was soft with exit of the PD catheter placed in the lower right abdomen. The exit site was clear. The patient's body temperature was $36.6^{\circ} \mathrm{C}$, with

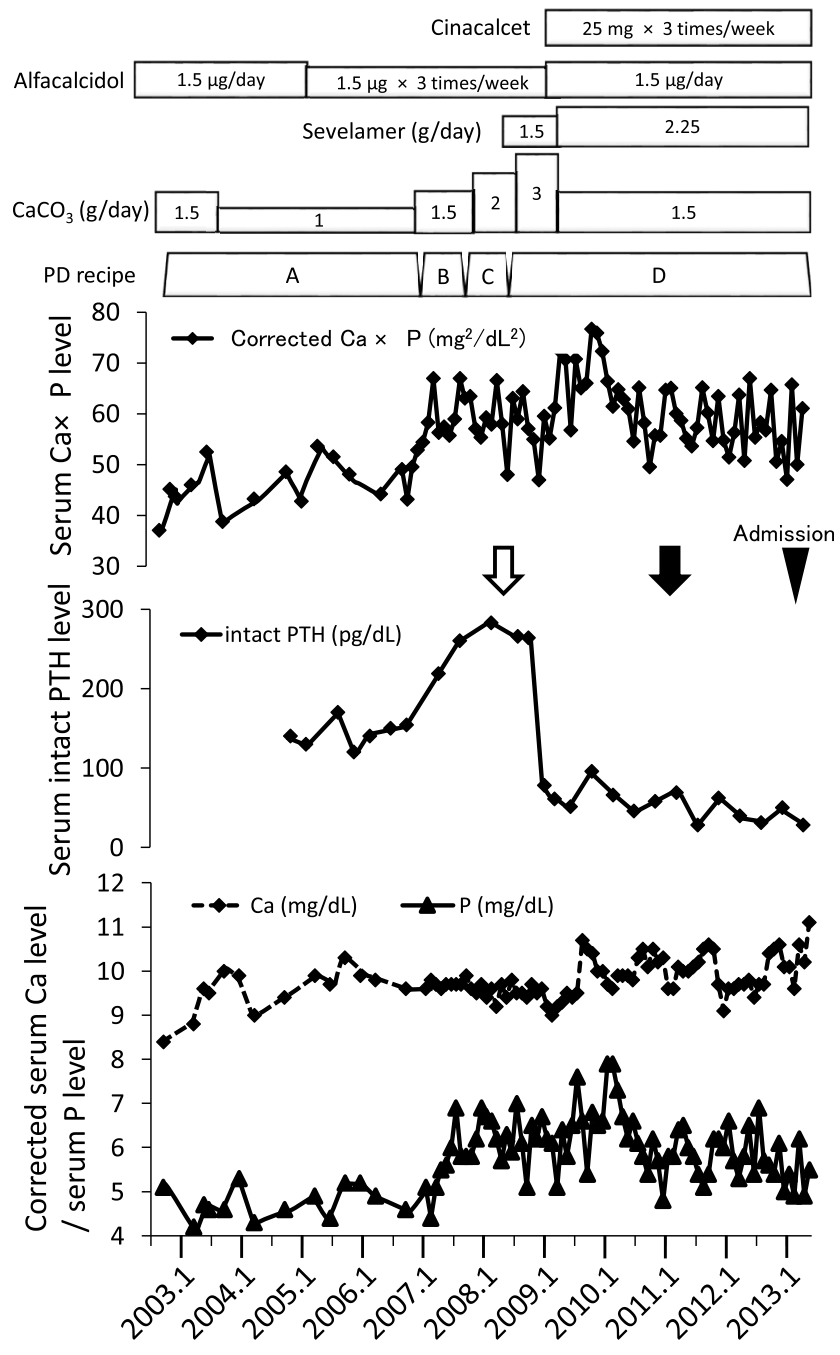

Figure 1. Trends in the serum levels of calcium (Ca), phosphate (P) and intact parathyroid hormone (iPTH) following the administration of phosphate binders and the peritoneal dialysate regimen. This figure shows the clinical course and laboratory data for calcium and phosphate metabolism starting in 2002, the beginning of peritoneal dialysis (PD) therapy. The administration of cinacalcet hydrochloride (cinacalcet), alfacalcidol, phosphate binders, such as calcium carbonate $\left(\mathrm{CaCO}_{3}\right)$ and sevelamer hydrochloride (sevelamer), is shown in the top bars of the figure. The doses are indicated in the frames. The arrows show the timing of the echocardiographic examinations and the arrowhead indicates the timing of admission for surgery. The PD recipe was changed as follows:A) $1.5 \mathrm{~L}$ of $1.5 \% \times 3$ times in the daytime and once overnight. B) $1.5 \mathrm{~L}$ of $1.5 \% \times 4$ times in the daytime and once overnight. C) $1.5 \mathrm{~L}$ of $1.5 \%$ or $2.5 \% \times 4$ times in the daytime and $1.5 \mathrm{~L}$ of icodextrin solution overnight. D) $1.5 \mathrm{~L}$ of $2.5 \% \times 3$ times in the daytime and $1.5 \mathrm{~L}$ of icodextrin solution overnight.

a blood pressure of $137 / 66 \mathrm{mmHg}$ and heart rate of 60 beats/min with a sinus rhythm. The clinical laboratory data for the blood and serum were as follows: white blood cell count, $4.6 \times 10^{3} / \mu \mathrm{L}$; red blood cell count, $340 \times 10^{4} / \mu \mathrm{L}$; hemoglobin, $9.0 \mathrm{~g} / \mathrm{dL}$; hematocrit, $27.9 \%$; platelet count, $15.2 \times$ $10^{4} / \mu \mathrm{L}$; C-reactive protein, $0.08 \mathrm{mg} / \mathrm{dL}$; total protein, $6.2 \mathrm{~g} /$ 
Table 2. Summary of D/P Creatinine of Peritoneal Equilibration Test and of Dialysis Efficacies

\begin{tabular}{llll}
\hline Year & D/P Creatinine & Kt/V (L/week) & Creatinine clearance(L/week) \\
\hline 2004 & 0.60 & $1.58(\mathrm{Dia})+1.37($ Res $)$ & $34.23(\mathrm{Dia})+47.15($ Res $)$ \\
2011 & 0.63 & $2.04(\mathrm{Dia})$ & $45.16(\mathrm{Dia})$ \\
2012 & 0.65 & $2.12(\mathrm{Dia})$ & $43.53(\mathrm{Dia})$ \\
\hline
\end{tabular}

D/P: dialysate-to-plasma creatinine concentration ratio, Dia: dialysis, Res: residual renal function.

Residual renal function was lost in 2011 and 2012.

$\mathrm{dL}$; albumin, $3.3 \mathrm{~g} / \mathrm{dL}$; glutamic oxaloacetic transaminase, $10 \mathrm{IU} / \mathrm{L} ;$ glutamic pyruvic transaminase, $6 \mathrm{IU} / \mathrm{L} ; \gamma$ glutamyl transpeptidase, $20 \mathrm{IU} / \mathrm{L}$; total cholesterol, $114 \mathrm{mg} / \mathrm{dL}$; highdensity lipoprotein cholesterol, $39 \mathrm{mg} / \mathrm{dL}$; low-density lipoprotein cholesterol, $51 \mathrm{mg} / \mathrm{dL}$; choline esterase, $271 \mathrm{IU} / \mathrm{L}$; total bilirubin, $0.3 \mathrm{mg} / \mathrm{dL}$; lactate dehydrogenase, $294 \mathrm{IU} / \mathrm{L}$; alkaline phosphatase, $702 \mathrm{IU} / \mathrm{L}$; creatinine kinese, $121 \mathrm{IU} / \mathrm{L}$; blood urea nitrogen, $76.0 \mathrm{mg} / \mathrm{dL}$; creatinine, $9.83 \mathrm{mg} / \mathrm{dL}$; sodium, $131 \mathrm{mEq} / \mathrm{L}$; potassium $3.4 \mathrm{mEq} / \mathrm{L}$; uric acid 7.6 $\mathrm{mg} / \mathrm{dL}$; corrected calcium, $10.82 \mathrm{mg} / \mathrm{dL}$; phosphate, $4.3 \mathrm{mg} /$ dL; glycosylated hemoglobin (NGSP), 8.2\%; iPTH, 28 pg/ $\mathrm{mL}$; activated partial thromboplastin time, 34 seconds (97.7\% compared to healthy controls); and prothrombin time (PT), 12.9 seconds (103.0\% compared to healthy controls). The data obtained from a blood gas analysis (room air) were as follows: $\mathrm{pH}, 7.397 ; \mathrm{pO}_{2}, 68.5 \mathrm{mmHg} ; \mathrm{pCO}_{2}, 40.9 \mathrm{mmHg}$; $\mathrm{cHCO}_{3}, 24.7 \mathrm{mmHg}$; and actual base excess, $0.4 \mathrm{mmol} / \mathrm{L}$. The electrocardiographic findings remained within the normal limits. Chest radiography showed enlargement of the heart, with a cardiothoracic ratio of approximately $60 \%$. On admission, simple thoracoabdominal computed tomography (CT) showed severe calcification of the aorta far from the level of bifurcation of the renal and coronary arteries; however, no significant ectopic calcification was noted around the subcutaneous soft tissues or joints. In addition, echocardiography confirmed a hyperechoic movable mass adherent to the mitral valve (Fig. 2a, b). The detailed echocardiographic results are shown in Table 3. On day 3 after admission, excision of the intracardiac tumor was performed. Intraoperatively, the tumor was seen to arise from a calcified area on the posterior wall of the left atrium, with gelatinlike material adhering around the tumor (Fig. 2c). The maximum diameter of the resected tumor was approximately 1 cm (Fig. 2d). A culture of the gelatin-like material yielded negative results, while a pathological examination of the tumor revealed a hypocellular, fibrous mass without evidence of malignancy, although the lesion was accompanied by severely calcified lesions (Fig. 2e, f). The final diagnosis was therefore CAT. The patient was subsequently maintained on HD in order to accurately control her body fluids for approximately one week after the operation, after which she quickly recovered. She was returned to PD therapy in accordance with her strong request on day 12 after admission and was discharged from the hospital on day 20.

\section{Discussion}

CAT is a rare cardiac tumor first proposed in 1997 by Reynolds et al. based on 11 cases (1). The background factors of CAT cases have been shown to involve various diseases, including malignancy, systemic lupus erythematosus and ischemic heart disease, with ESRD reported in one case (1).

Although the etiology of CAT remains unclear, a thrombotic state has been suggested to induce the development of CAT in association with hypercoagulation and/or degenerated thrombosis (1). Disorders of calcium and phosphate metabolism are also considered to be potential causes of CAT (2). Although a small number of cases of CAT have been reported in ESRD patients on HD (2-6), no cases involving ESRD patients on PD have been reported. We herein provided the first description of the onset of CAT in an ESRD patient on long-term PD.

ESRD patients usually exhibit bone mineral disorders, and mitral annulus calcification (MAC) and tumoral calcinosis have been reported to be complications of such patients, with increasing corrected calcium-phosphate product levels, hyperparathyroidism and low turnover of bone $(9,10)$. In the present case, the patient had received PD therapy for 11 years, and high calcium-phosphate product and serum iPTH levels were observed prior to the formation of CAT (Fig. 1). Therefore, the increased serum corrected calcium and phosphate levels and iPTH value may have facilitated the development of CAT. In addition, the serum phosphate level is consistently high in patients on PD, whereas it temporarily decreases during every HD session. Patients on PD may therefore be more affected by an increased calciumphosphate product level than patients on HD. However, the relationships between CAT formation and the calciumphosphate product level or duration of dialysis remain unclear. In PD patients with a high level of serum calcium, the use of a PD solution with a low calcium concentration appears to improve the calcium balance and may have been a suitable choice in the present case at the previous hospital when the serum level of corrected calcium was high. However, the improve in both the serum calcium and phosphate levels was not sufficient to persuade the patient to change from a PD solution with a standard calcium concentration to a solution with a low calcium concentration, according to a physician from the previous hospital. In addition, the patient 

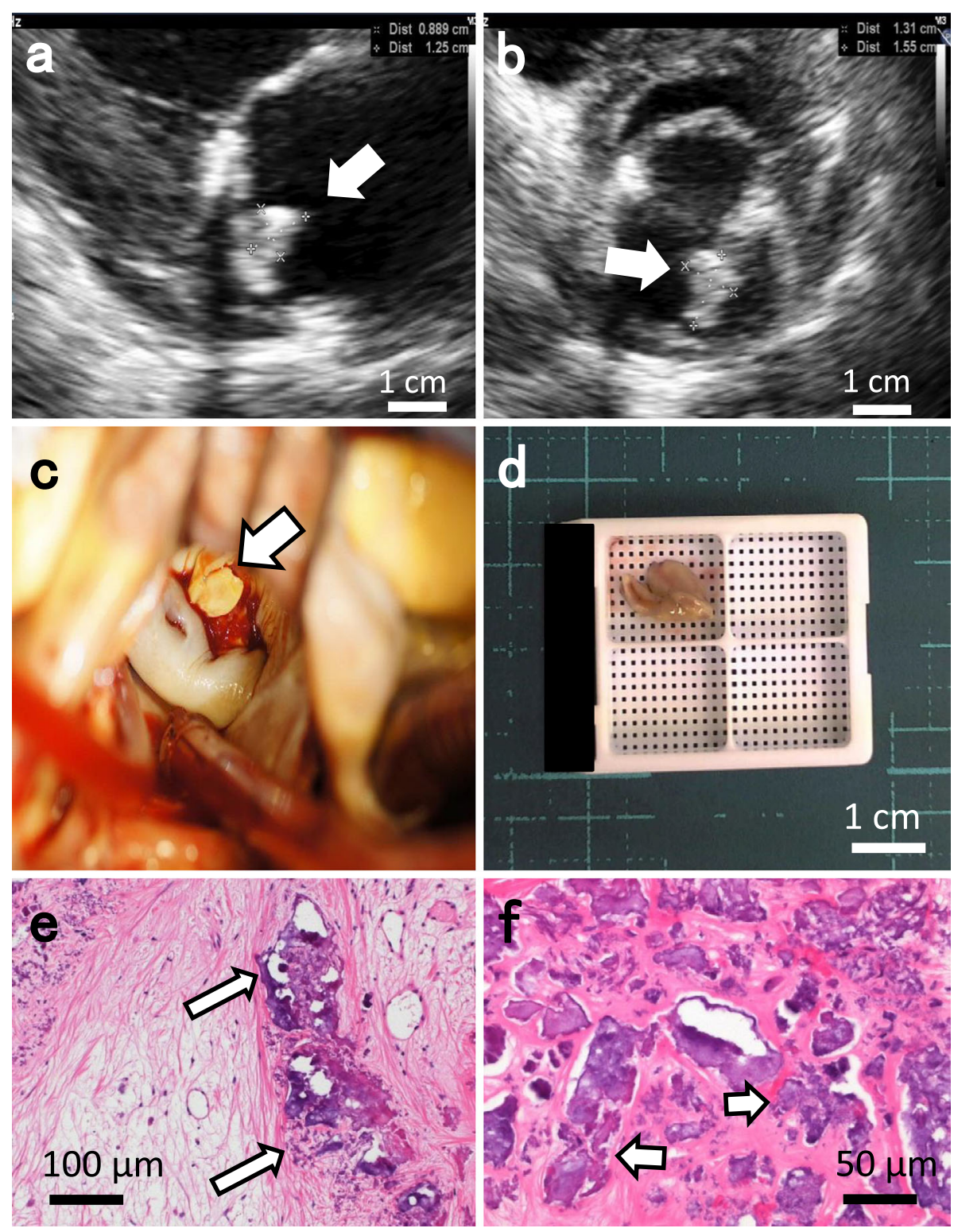

Figure 2. Echocardiography findings on admission, the intraoperative appearance of the tumor and light microscopy findings. Echocardiography performed in early 2013 upon admission to our hospital. a) Long-axis view. b) Short-axis view. The white arrows show a highly echoic, mobile mass adherent to the mitral valve. c) The arrow shows the tumor in the left atrium. d) The resected tumor. e, f) Findings of light microscopy with Hematoxylin and Eosin staining. The arrows indicate the calcified lesion [original magnification $\times 200(e)$ and $\times 400(f)]$.

did not show good compliance with dietary advice related to calcium and phosphate metabolism, and an assessment of the serum level of glycosylated hemoglobin as a possible factor inducing ectopic calcification showed her blood sugar control to generally be adequate at the previous hospital, although poor glycemic control may have been related to the progression of vascular calcification (11). Furthermore, thrombosis may also be a cause of CAT formation, as ESRD patients may have coagulation abnormalities (12) and/or receive anticoagulant or antiplatelet agents (1). Previous reports of CAT in ESRD patients on HD therapy may have been associated with the regular use of anticoagulants during HD. Another possibility regarding the onset of CAT is the opportunity for bacteremic events, such as endocardi- tis, to induce the development of organized thrombi in ESRD patients, who are generally immunocompromised. However, the current patient had no history of treatment with anticoagulant or antiplatelet agents and had not experienced any bacteremic events other than peritonitis. Importantly, the asymptomatic CAT was only identified on routine echocardiography in this case. We thus consider routine echocardiography to be important for making an early diagnosis of CAT, as, in this and previous cases, CAT has been shown to develop rapidly unaccompanied by symptoms (6). CAT may originate in any of the four chambers of the heart (1), although the development of this condition in patients on HD has been reported to be related to MAC (3, 4, 6). However, diagnosing CAT based only on the 
Table 3. Summary of Echocardiographic Findings in June 2008 and May 2013

\begin{tabular}{|c|c|c|c|}
\hline & Normal range & June 2008 & May 2013(on admission) \\
\hline $\mathrm{AOD}(\mathrm{mm})$ & $20-37$ & 31.1 & 29.0 \\
\hline LAD (mm) & $19-40$ & 45.1 & 48.8 \\
\hline LVDd (mm) & $40-55$ & 49.4 & 49.7 \\
\hline LVDs (mm) & $30-45$ & 30.1 & 30.2 \\
\hline IVSTd (mm) & $7-11$ & 13.4 & 15.6 \\
\hline LVPWTd (mm) & $7-11$ & 12.2 & 10.4 \\
\hline $\mathrm{EF}(\%)$ & $>55$ & not determined & 69.6 \\
\hline FS $(\%)$ & $>28$ & 39.1 & 39.2 \\
\hline \multicolumn{2}{|c|}{ Left ventricular wall motion } & within normal limits & within normal limits \\
\hline \multicolumn{2}{|c|}{ Mitral regurgitation } & none & moderate \\
\hline \multicolumn{2}{|l|}{ Aortic regurgitation } & none & none \\
\hline \multicolumn{2}{|l|}{ Tricuspid regurgitation } & mild & moderate \\
\hline \multicolumn{2}{|l|}{ Aortic stenosis } & none & none \\
\hline E/A ratio & $>1$ & 0.83 & 0.9 \\
\hline DcT (msec) & $>150$ & 168 & 174 \\
\hline \multicolumn{2}{|l|}{ Mass lesion in LA } & not detected & $9 \times 13 \mathrm{~mm}$ \\
\hline \multicolumn{2}{|l|}{ Valve calcification } & mild in aortic valve & mild in mitral valve \\
\hline \multicolumn{4}{|c|}{$\begin{array}{l}\text { AOD: aortic orifice diameter, LAD: left atrial dimension, LVDd: left ventricular end-diastolic } \\
\text { dimension, LVDs: left ventricular end-systolic diamesion, IVSTd: end-diastolic interventricular } \\
\text { septal wall thickness, LVPWTd: left ventricular posterior wall end-diastolic thickness, EF: ejection } \\
\text { fraction, FS: fractional shortening, E/A ratio: a ratio of peak mitral E wave velocity to peak mitral A }\end{array}$} \\
\hline
\end{tabular}

presence of MAC is difficult, as MAC is not an uncommon finding in ESRD patients. Furthermore, it can be very difficult to differentiate CAT from vegetation, myxoma and thrombosis with calcification in both in non-ESRD and ESRD patients, and surgical resection is necessary to achieve a definitive diagnosis and prevent embolism in such cases $(7,8)$.

The present study is the first report of CAT in a PD patient. Routine echocardiography is useful for screening to achieve an early diagnosis of CAT in ESRD patients and prevent unexpected complications. Although the mechanisms underlying the development of CAT are unclear, controlling the levels of calcium and phosphate is important for preventing the development of CAT in ESRD patients.

The authors state that they have no Conflict of Interest (COI).

\section{References}

1. Reynolds C, Tazelaar HD, Edwards WD. Calcified amorphous tumor of the heart (cardiac CAT). Hum Pathol 28: 601-606, 1997.

2. Vlasseros I, Katsi V, Tousoulis D, et al. Visual loss due to cardiac calcified amorphous tumor: a case report and brief review of the literature. Int J Cardiol 152: e56-e57, 2011.

3. Fujiwara $M$, Watanabe $H$, Iino $T$, et al. Two cases of calcified amorphous tumor mimicking mitral valve vegetation. Circulation 125: e432-e434, 2012.

4. Kubota H, Fujioka Y, Yoshino H, et al. Cardiac swinging calcified amorphous tumors in end-stage renal failure patients. Ann Thorac Surg 90: 1692-1694, 2010.

5. Sousa JS, Tanamati C, Marcial MB, Stolf NA. Calcified amorphous tumor of the heart: case report. Rev Bras Cir Cardiovasc 26: 500-503, 2011.

6. Kawata T, Konishi H, Amano A, Daida H. Wavering calcified amorphous tumour of the heart in a haemodialysis patient. Interact Cardiovasc Thorac Surg 16: 219-220, 2013.

7. Rytand DA, Lipsitch LS. Clinical aspects of calcification of the mitral annulus fibrosus. Arch Intern Med 78: 544-564, 1946.

8. Benjamin EJ, Plehn JF, D'Agostino RB, et al. Mitral annular calcification and the risk of stroke in an elderly cohort. N Engl J Med 327: 374-379, 1992.

9. Asselbergs FW, Mozaffarian D, Katz R, et al. Association of renal function with cardiac calcifications in older adults: the cardiovascular health study. Nephrol Dial Transplant 24: 834-840, 2009.

10. Olsen KM, Chew FS. Tumoral calcinosis: pearls, polemics, and alternative possibilities. Radiographics 26: 871-885, 2006.

11. Ishimura E, Okuno S, Taniwaki H, Kazu A. Different risk factors for vascular calcification in end-stage renal disease between diabetics and nondiabetics: the respective importance of glycemic and phosphate control. Kidney Blood Press Res 31: 10-15, 2008.

12. Rabelink TJ, Zwaginga JJ, Koomans HA, Sixma JJ. Thrombosis and hemostasis in renal disease. Kidney Int 46: 287-296, 1994.

(C) 2015 The Japanese Society of Internal Medicine http://www.naika.or.jp/imonline/index.html 